\title{
Quantum ergodicity for a class of mixed systems
}

\author{
Jeffrey Galkowski
}

\begin{abstract}
We examine high energy eigenfunctions for the Dirichlet Laplacian on domains where the billiard flow exhibits mixed dynamical behavior. (More generally, we consider semiclassical Schrödinger operators with mixed assumptions on the Hamiltonian flow.) Specifically, we assume that the billiard flow has an invariant ergodic component, $U$, and study defect measures, $\mu$, of positive density subsequences of eigenfunctions (and, more generally, of almost orthogonal quasimodes). We show that any defect measure associated to such a subsequence satisfies $\left.\mu\right|_{U}=\left.c \mu_{L}\right|_{U}$, where $\mu_{L}$ is the Liouville measure. This proves part of a conjecture of Percival [18].
\end{abstract}

Mathematics Subject Classification (2010). 58J51, 58J32.

Keywords. Quantum ergodicity, mixed dynamics, manifold with boundary.

\section{Introduction}

The distribution of eigenfunctions over phase space in the semiclassical limit is an important object of study in the theory of quantum chaos. The fundamental result is a quantum ergodicity theorem of Shnirelman [19], Zelditch [21], and Colin de Verdière [5] which states that, for classically ergodic systems, high energy eigenfunctions distribute uniformly in phase space. Since we will study domains with boundary, we note that Gérard and Leichtnam [7] and Zelditch and Zworski [22] generalized quantum ergodicity to that case.

Some progress has been made toward understanding semiclassical limits of eigenfunctions for systems with dynamical behavior that is not completely ergodic. Marklof and O'Keefe [13] examine separated phase spaces for certain maps. More recently, Marklof and Rudnick [14] have made further strides towards an understanding of quantum behavior for mixed phase space. In particular, they prove that, for rational polygons, the eigenfunctions of the Dirichlet Laplacian equidistribute in configuration space. We use [14] as inspiration for further work on semiclassical limits for systems with mixed dynamical behavior. 
We examine systems whose phase spaces have invariant subsets, $U$, such that the flow restricted to $U$ is ergodic and the Liouville measure of $U$ is positive. In particular, let $(M, g)$ be a smooth Riemannian manifold of dimension $d$ with a piecewise smooth boundary, $\partial M$. Then $M \subset \tilde{M}$, where $\tilde{M}$ is a manifold without boundary to which $g$ extends smoothly, and $\partial M=\bigcup_{j=1}^{J} N_{j}$ where $N_{j}$ are smooth embedded hypersurfaces in $\tilde{M}$. Define $\partial^{o} M \subset \partial M$ to be the open set of all points where the boundary is smooth. Then the complement $\partial M \backslash \partial^{o} M$ has measure zero.

We consider

$$
P(h)=-h^{2} \Delta+V
$$

with Dirichlet boundary conditions and let $p(x, \xi)$ be its principal symbol. Since $p(x, \xi)$ is smooth up to the boundary, we can extend it smoothly to $T^{*} \tilde{M}$. We assume that

$$
\left.d p\right|_{p^{-1}(E)} \neq 0, \quad E \in[a, b],
$$

and

$$
x \in \partial^{o} M, V(x)=E \Longrightarrow d V \notin N_{x}^{*} \partial^{o} M .
$$

so that $p^{-1}(E)$ and $T_{\partial^{o} M}^{*} M$ intersect transversally.

We then write

$$
p^{-1}(E) \cap T_{\partial^{o} M}^{*} M=\Omega_{E}^{+} \sqcup \Omega_{E}^{-} \sqcup \Omega_{E}^{0},
$$

where $(x, \xi)$ lies in $\Omega_{E}^{+}$if the vector $H_{p} x \in T \tilde{M}$ points outside of $M$, in $\Omega_{E}^{-}$if it points inside $M$, and $\Omega_{E}^{0}$ if it is tangent to $\partial M$. The set $\Omega_{E}^{0}$ contains the glancing covectors and, under (1.2), has measure zero in $p^{-1}(E) \cap T_{\partial^{o}}^{*} M$.

We define the broken Hamiltonian flow as follows. (see, for example, [6], Appendix A). We denote this flow by

$$
\varphi_{t} \stackrel{\text { def }}{=} \exp \left(H_{p} t\right)
$$

Assume without loss of generality that $t>0$. We consider $\exp \left(t H_{p}\right)(x, \xi)$ defined on $T^{*} \tilde{M}$, and let $t_{0}$ be the first nonnegative time when $\exp \left(t H_{p}\right)(x, \xi)$ hits the boundary. If this happens at a non-smooth point of the boundary, or if $\exp \left(t_{0} H_{p}\right)(x, \xi) \in \Omega_{E}^{0}$, then the flow cannot be extended past $t=t_{0}$. Otherwise, $\exp \left(t_{0} H_{p}\right)(x, \xi) \in \Omega_{E}^{+}$and there exists unique $\left(x_{0}, \xi_{0}\right) \in \Omega_{E}^{-}$such that the natural projections of $\exp \left(t_{0} H_{p}\right)(x, \xi)$ and $\left(x_{0}, \xi_{0}\right)$ onto $T^{*} \partial M$ are the same. We then define $\varphi_{t}$ inductively, by putting $\varphi_{t}(x, \xi)=\exp \left(t H_{p}\right)(x, \xi)$ for $0<t<t_{0}$ and $\varphi_{t}(x, \xi)=\varphi_{t-t_{0}}\left(x_{0}, \xi_{0}\right)$ for $t>t_{0}$. Define for $T>0$, the set

$$
\mathcal{B}_{T} \subset T^{*} M \cap p^{-1}([a, b])
$$

to be the closed set of all $(x, \xi)$ such that $\varphi_{t}(x, \xi)$ intersects a glancing point for some $t \in[-T, T]$. Then, as shown in [22], Lemma $1, \mathcal{B}_{T}$ has measure zero in $p^{-1}(E)$. 
In many cases, quasimodes with specified concentration properties are easier to construct than corresponding eigenfunctions. One example of this is Hassell's use of concentrating quasimodes in [8] to show non quantum unique ergodicity in some domains with ergodic billiard flow (for another, see Section 5). Other connections between quasimodes and quantum ergodicity have been explored in [1], [12], and [20]. The utility of quasimodes motivates us to generalize existing quantum ergodicity results to that setting (Section 2). Although this is a natural generalization and not unexpected, it does not seem to be available in the literature.

We make the following definitions (with the obvious analog in the homogeneous setting under the rescaling $h=\lambda^{-1}$ ).

Definition 1. A positive density set of quasimodes for $P$ on $[a, b], 0 \leq a<b$ is a collection

$$
\left\{\left(\psi_{j}, E_{j}\right), j=1, \ldots\right\}
$$

satisfying,

(1) $\left\|\psi_{j}\right\|_{L^{2}}=1$,

(2) $\left\|\left(P-E_{j}\right) \psi_{j}\right\|_{L^{2}}=o\left(h^{2 d+1}\right)$,

(3) $\left|\left\langle\psi_{j}, \psi_{k}\right\rangle\right|=o\left(h^{2 d}\right)$ for $j \neq k$,

(4) $\left|\left\{E_{j} \in[a, b]\right\}\right| \geq c h^{-d}, c>0$.

Definition 2. Let $E_{j}, j=1, \ldots$ be all the eigenvalues of $P$ with multiplicity. A complete set of quasimodes for $P$ is a collection $\left\{\psi_{j}, j=1, \ldots\right\}$ satisfying

(1) $\left\|\psi_{j}\right\|_{L^{2}}=1$,

(2) $\left\|\left(P-E_{j}\right) \psi_{j}\right\|_{L^{2}}=o\left(h^{2 d+1}\right)$,

(3) $\left|\left\langle\psi_{j}, \psi_{k}\right\rangle\right|=o\left(h^{2 d}\right)$, for $j \neq k$.

Remarks 1. (1) Notice that the set of all eigenfunctions is a complete set of quasimodes in the sense of Definition 2, in particular, a positive density set of quasimodes for $P$ on $[a, b]$. Hence, our results apply to eigenfunctions.

(2) The discrepancies for these quasimodes are much smaller than the mean spectral density given by the Weyl law and are necessary so that Hilbert-Schimidt norms can be accurately expressed in terms of quasimodes.

(3) Note that, although it is often easy to construct positive density sets of quasimodes with specified concentration properties, it is difficult to construct complete sets of quasimodes with the same. However, the proofs of our results are simplified by using complete sets of quasimodes. To avoid these difficulties, we formulate our results in terms of positive density sets of quasimodes. and prove in Lemma 4 that it is enough to consider complete sets of quasimodes. 
Under assumptions (1.1), (1.2), and

$$
\varphi_{t} \text { is ergodic on } p^{-1}(E), \quad E \in[a, b]
$$

we have the following analog of quantum ergodicity for a positive density set of quasimodes for $P$. For a semiclassical pseudodiffential operator of order $m$, we write $B \in \Psi_{h}^{m}$, and denote its semiclassical symbol, $\sigma_{h}(B)$ (see, for example, [23], Section 14.2, for definitions).

Theorem 1. Suppose that $(M, g)$ is a compact manifold with a piecewise smooth boundary and (1.3) holds. Let $\left\{\left(\psi_{j}, E_{j}\right), j=1, \ldots\right\}$ be a positive density set of quasimodes of the Dirichlet realization of $P$ on $[a, b]$ for $0<a<b$. Then there exist a family of subsets

$$
\Lambda(h) \subset\left\{\psi_{j} \mid a \leq E_{j} \leq b\right\}
$$

of full density such that if $\left\{\psi_{k}\right\} \subset \Lambda(h)$ is a sequence with unique defect measure $\mu$ then there is an $E \in[a, b]$ such that

$$
\mu \equiv\left(\mu_{E}\left(p^{-1}(E)\right)\right)^{-1} \mu_{E} .
$$

Here a defect measure for a sequence $\psi_{j}$ is the weak limit of the semiclassical measures $\mu_{j}$ associated to $\psi_{j}$ (See, for example, [23], Chapter 5, for details.)

Remarks 2. (1) An equivalent way of formulating this theorem is that for all $\psi_{j} \in$ $\Lambda(h)$ and $B \in \Psi_{h}^{0}\left(M^{o}\right)$ with symbol, $\sigma_{h}(B)$, compactly supported away from $\partial M$,

$$
\left|\left\langle B \psi_{j}, \psi_{j}\right\rangle-f_{p^{-1}\left(E_{j}\right)} \sigma_{h}(B) d \mu_{E_{j}}\right| \longrightarrow 0 .
$$

(2) Note that Theorem 1 applies to positive density subset of eigenfunctions in energy bands $E_{j} \in[a, b]$, while that in [9] is stronger and applies to such subsets of energy shells $E_{j} \in[a-C h, a+C h]$. However this does not apply to manifolds with boundary and such an energy shell quantum ergodicity theorem is not known in that case; see [9]. It would be interesting to obtain such a result.

In Section 3, we relax the dynamical assumption (1.3) and instead make the mixed dynamical hypotheses:

$$
\text { there exists } U \subset p^{-1}([a, b]) \text { such that }\left\{\begin{array}{l}
\mu_{E}\left(U \cap p^{-1}(E)\right)>0, \\
\mu_{E}\left(\partial U \backslash U \cap p^{-1}(E)\right)=0,
\end{array}\right.
$$

for $E \in[a, b]$, where $\mu_{E}$ is the Liouville measure on $p^{-1}(E)$ and

$$
\left\{\begin{array}{l}
\varphi_{t} \text { is ergodic on } U \cap p^{-1}(E), \quad E \in[a, b] \\
\varphi_{t}(U)=U
\end{array}\right.
$$


Remark 3. Hypotheses (1.4) include the case where $U$ is closed with a boundary of positive measure.

In this case, we have the following result:

Theorem 2. Suppose that $(M, g)$ is a compact manifold with a piecewise smooth boundary, and that (1.1), (1.2), (1.4), and (1.5) hold. Let $\left\{\left(\psi_{j}, E_{j}\right), j=1, \ldots\right\}$ be a positive density set of quasimodes of the Dirichlet realization of $P$ on $[a, b]$, $0<a<b$. Then there exist a family of subsets

$$
\Lambda(h) \subset\left\{\psi_{j} \mid a \leq E_{j} \leq b\right\}
$$

of full density such that if $\left\{\psi_{k}\right\} \subset \Lambda(h)$ is a further subsequence such that $\left\{\psi_{k}\right\}$ has unique defect measure, $\mu$, then there exist $E \in[a, b]$ and $c$ such that

$$
\operatorname{supp} \mu \subset p^{-1}(E)
$$

and

$$
\left.\left.\mu\right|_{U \cap p^{-1}(E)} \equiv c \mu_{E}\right|_{U \cap p^{-1}(E)}
$$

Remark 4. Although Theorem 1 can be seen as a corollary of Theorem 2 , the proof of Theorem 1 is essential to that of Theorem 2 and, in addition, presenting the direct proof is useful to demonstrate the new ideas involved in obtaining Theorem 2.

In Section 4, we specialize to the case $P=-h^{2} \Delta$ and pass from the semiclassical quantization to the Kohn-Nirenberg calculus. For a homogeneous pseudodifferential operator of order $m$, we write $B \in \Psi^{m}$ and denote its symbol, $\sigma(B)$ (see, for example, [10], Chapter 18, for definitions).

Theorem 3. Suppose that $(M, g)$ is a compact manifold with a piecewise smooth boundary and (1.3) holds. Let $\left\{\left(\psi_{j}, E_{j}\right), j=1, \ldots\right\}$ be a positive density set of quasimodes of the Dirichlet realization of $-\Delta$ on $[0,1]$. Then, there is a full density subsequence $\psi_{j_{k}}$ such that for all pseudodifferential operators $B \in \Psi^{0}\left(M^{o}\right)$ with symbols compactly supported away from $\partial M$,

$$
\left\langle B \psi_{j_{k}}, \psi_{j_{k}}\right\rangle \longrightarrow \int_{S^{*} M} \sigma(B) d \mu_{L} .
$$

Remark 5. Although the passage from Theorem 1 to 3 is known in the case of eigenfunctions and is not unexpected for quasimodes, we include it here because neither the result for quasimodes nor the passage from the semiclassical to the homogeneous case seem to appear in the literature.

We also specialize Theorem 2 to the homogeneous setting as follows. 
Theorem 4. Suppose that $(M, g)$ is a compact manifold with a piecewise smooth boundary and that there exists

$$
U \subset S^{*} M
$$

such that

(1) $\mu_{L}(U)>0$,

(2) $\mu_{L}(\partial U \backslash U)=0$,

(3) $\varphi_{t}$ is ergodic on $U$,

(4) $\varphi_{t}(U)=U$.

Let $\left\{\left(\psi_{j}, E_{j}\right), j=1, \ldots\right\}$ be a positive density set of quasimodes of the Dirichlet realization of $-\Delta$ on $[0,1]$. Then there is a full density subsequece of $\psi_{j}$ such that if any further subsequence $\psi_{j_{k}}$ has unique defect measure $\mu$, then $\left.\mu\right|_{U}=\left.c \mu_{L}\right|_{U}$ for some c.

Remark 6. In the homogeneous and boundaryless case, Riviére [17] recently proved a theorem on accumulation points of semiclassical measures that gives similar results for separated phase spaces. He also provides examples of separated phase spaces for the geodesic flow on boundaryless manifolds.

Percival [18] made conjectures on eigenfunctions that were numerically verified by Barnett and Betcke in [2] in the case of mushroom billiards. In particular, Percival used the quantum-classical correspondence principle to state that most eigenfunctions concentrate either entirely in the ergodic portion of phase space or in the integrable portion and, moreover, that the eigenfunctions concentrating in the ergodic portion of phase space spread uniformly over that piece of phase space. Although we are not able to show the dichotomy between integrable and chaotic eigenfunctions, Theorems 4 and 2 provide rigorous proofs of the fact that most eigenfunctions spread uniformly in the ergodic portions of phase space.

Finally, in Section 5, we apply our results to the Dirichlet Laplacian for mushroom billiards. Figure 1 shows two billiards trajectories, one in the ergodic region and one in the integrable region. (For a complete description of the billiard map on mushrooms see [3].)

Acknowledgements. The author would like to thank Maciej Zworski for valuable discussion, Stéphane Nonnenmacher and Zeév Rudnick for pointing him toward mushroom billiards and [14], Semyon Dyatlov for advice on boundary value problem quantum ergodicity, Alex Barnett for allowing him to use the images in Figure 2 and referring him to [18], and the anonymous reviewer for many helpful comments. The author is grateful to the Erwin Schrödinger Institue for support during the Summer School on Quantum Chaos and to the National Science Foundation for support under the National Science Foundation Graduate Research Fellowship Grant No. DGE 1106400, and for support during the Erwin Schrödinger Institute's Summer School on Quantum Chaos. 

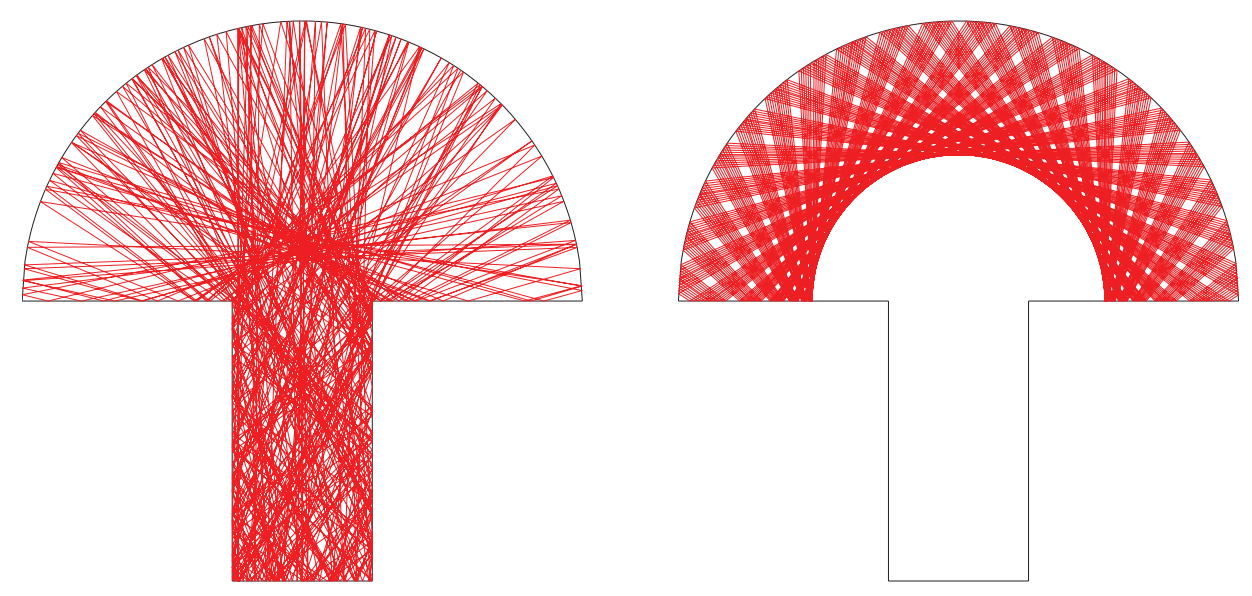

Figure 1. We show two billiards trajectories in a Bunimovich mushroom [3] with semicircular hat of radius 1 and centered base of width $1 / 2$ and height 1 . On the left, we have a trajectory in the ergodic portion of phase space. On the right, we have one in the integrable portion.
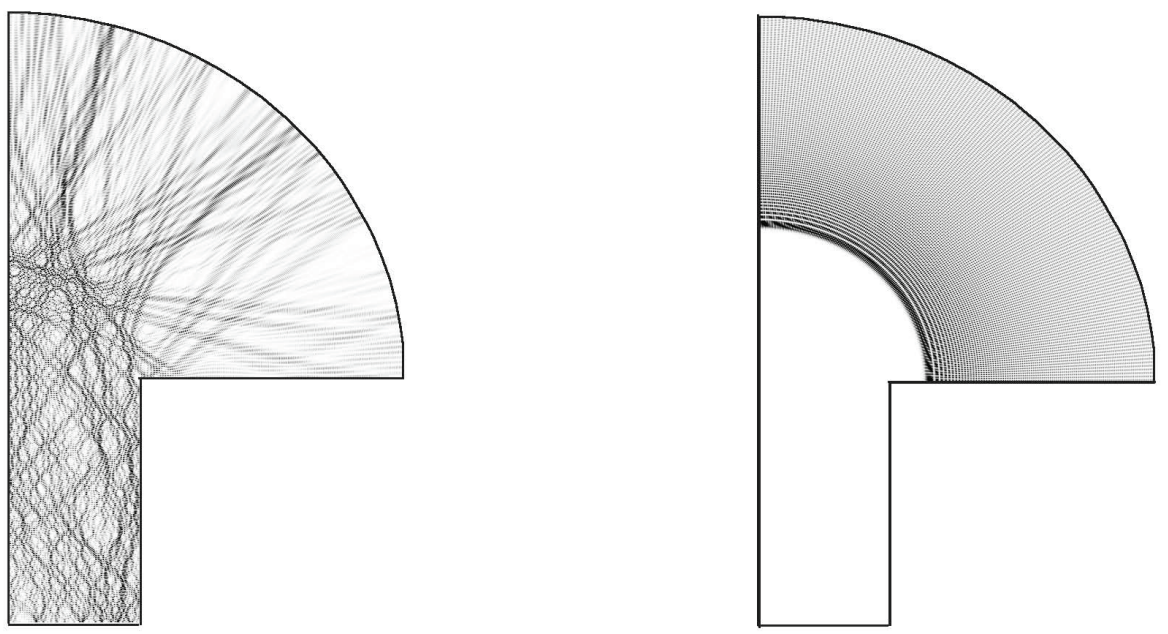

Figure 2. We show two high energy eigenfunctions for the Laplacian in a Bunimovich mushroom [3]. The left hand eigenfunction spreads uniformly through the ergodic portion of phase space, and the right hand eigenfunction concentrates in the integrable portion. (Images courtesy of A. Barnett and T. Betcke [2]). 


\section{Quantum ergodicity for quasimodes}

In this section, we demonstrate how to adapt quantum ergodicity results for eigenfunctions to positive density sets of quasimodes.

We will need the following lemmas relating various spectral quantities for operators to their corresponding expressions involving complete sets of quasimodes.

Lemma 1. Let $\left\{u_{k}, k=1, \ldots\right\}$ be the eigenfunctions of $P$ corresponding to $E_{k}$ and let $\left\{\psi_{j}, j=1, \ldots\right\}$ be a complete set of quasimodes for $P$. Then,

$$
u_{j}=\sum_{\left|E_{j}-E_{k}\right|<C h^{d+1}} c_{j k} \psi_{k}+o\left(h^{d}\right) .
$$

Proof. First, observe that, near an energy level $E$, there exists $\left(c_{1}(h), c_{2}(h)\right)$ with $\left|c_{1}(h)-c_{2}(h)\right| \geq c h^{d+1}$ such that

$$
\left(\left(E-c_{2}(h), E-c_{1}(h)\right) \cup\left(E+c_{1}(h), E+c_{2}(h)\right)\right) \cap\left\{E_{j}, j=1, \ldots\right\}=\emptyset .
$$

If this were false, then the spectrum of $P$ would violate the Weyl law.

Now, let

$$
\Lambda=\left\{\psi_{j} \mid E_{j} \in\left(E-c_{1}(h), E+c_{1}(h)\right)\right\}
$$

and $\Pi$ be the spectral projection onto $\left[E-c_{1}(h), E+c_{1}(h)\right]$. By the Weyl law, $|\Lambda| \leq C h^{-d}$. Therefore, by [11], Proposition 32.4,

$$
\operatorname{dim}(\Pi \operatorname{span}(\Lambda))=|\Lambda|,
$$

since

$$
o\left(h^{2 d}\right)+o\left(h^{2 d+1}\right) O\left(h^{-d-1}\right)=o\left(h^{d}\right) .
$$

But, rank $\Pi=|\Lambda|$. Hence, span $\Lambda=$ range $\Pi$ and the result follows from the almost orthogonality of $\psi_{j}$.

Lemma 2. Let $A$ be a Hilbert-Schmidt operator and $\left\{\psi_{j}, j=1, \ldots\right\}$ be a complete set of quasimodes for $P$ and $a<b$. Then,

$$
h^{d} \sum_{a \leq E_{j} \leq b}\left\|A \psi_{j}\right\|^{2}=h^{d}\left\|\Pi_{[a, b]} A\right\|_{H S}^{2}+o(1)
$$

and if $A$ is of trace class,

$$
h^{d} \sum_{a \leq E_{j} \leq b}\left\langle A \psi_{j}, \psi_{j}\right\rangle=h^{d} \operatorname{Tr} \Pi_{[a, b]} A+o(1) .
$$


Proof. First, let $\left(c_{j k}\right)=\left\langle u_{k}, \psi_{j}\right\rangle$. Then, by Lemma 1,

$$
u_{j}=\sum_{k} c_{j k} \psi_{k}+o\left(h^{d}\right) \quad \text { and } \quad \psi_{j}=\sum_{k} \overline{c_{j k}} u_{k},
$$

where all sums are taken over $k$ such that $\left|E_{k}-E_{j}\right|<C h^{d+1}$. Observe that

$$
u_{j}=\sum_{k} c_{j k} \psi_{k}+o\left(h^{d}\right)=\sum_{k, k^{\prime}} c_{j k} \overline{c_{k k^{\prime}}} u_{k^{\prime}}+o\left(h^{d}\right) .
$$

Hence, by the orthogonality of $u_{j}$,

$$
\sum_{k} c_{j k} \overline{c_{k k^{\prime}}}=\delta_{k j}+o\left(h^{d}\right) .
$$

Now,

$$
\begin{aligned}
h^{d} \sum_{a \leq E_{j} \leq b}\left\langle A \psi_{j}, A \psi_{j}\right\rangle & =h^{d} \sum_{j, k, k^{\prime}} c_{j k} \overline{c_{j k^{\prime}}}\left\langle A u_{k}, A u_{k}^{\prime}\right\rangle \\
& =h^{d} \sum_{k, k^{\prime}}\left(\delta_{k k^{\prime}}+o\left(h^{d}\right)\right)\left\langle A u_{k}, A u_{k^{\prime}}\right\rangle \\
& =h^{d}\left\|\Pi_{[a, b]} A\right\|_{H S}^{2}+h^{d} \sum_{k, k^{\prime}} o\left(h^{d}\right)\left\langle A u_{k}, A u_{k^{\prime}}\right\rangle \\
& =h^{d}\left\|\Pi_{[a, b]} A\right\|_{H S}^{2}+o(1)
\end{aligned}
$$

where the last equality follows from the fact that there are at most $O\left(h^{-d}\right)$ terms in each sum.

An analogous argument shows that (2.2) holds.

To prove Theorem 1, we need the following lemma similar to [6], Lemma A.2,

Lemma 3. Let $\chi \in C_{c}^{\infty}\left(M^{o}\right)$ with $0 \leq \chi \leq 1$. Then for $a^{\prime}<a<b<b^{\prime}$,

$$
\begin{aligned}
(2 \pi h)^{d} \sum_{E_{j} \in[a, b]} \int_{M}(1-\chi)\left|\psi_{j}\right|^{2} d \mathrm{Vol} & \\
\leq & \int_{T^{*} M \cap p^{-1}\left(\left[a^{\prime}, b^{\prime}\right]\right)}(1-\chi) d \mu_{\sigma}+o(1) \text { as } h \rightarrow 0 .
\end{aligned}
$$

Proof. This follows directly from [6], Lemma A.2, and Lemma 2 once we observe that

$$
\begin{aligned}
(2 \pi h)^{d} \sum_{E_{j} \in[a, b]} \int_{M}(1-\chi)\left|\psi_{j}\right|^{2} d \mathrm{Vol} & =(2 \pi h)^{d} \sum_{E_{j} \in[a, b]}\left\langle(1-\chi) \psi_{j}, \psi_{j}\right\rangle \\
& =(2 \pi h)^{d} \operatorname{Tr} \Pi_{[a, b]}(1-\chi)+o(1) .
\end{aligned}
$$


The following lemma allows us to take arbitrary collections of orthogonal quasimodes and complete them. Hence, we only have to prove results for complete sets of quasimodes and they follow for positive density sets.

Lemma 4. Let $\left\{\left(\psi_{j}, E_{j}\right), j=1, \ldots, J\right\}$ be a set of almost orthogonal quasimodes with

(1) $\left\|\psi_{j}\right\|_{L^{2}}=1$,

(2) $\left\|\left(P-E_{j}\right) \psi_{j}\right\|_{L^{2}}=o\left(h^{2 d+1}\right)$,

(3) $\left|\left\langle\psi_{j}, \psi_{k}\right\rangle\right|=o\left(h^{2 d}\right)$, for $j \neq k$.

Then, there exists

$$
\left\{\varphi_{k}, k=1, \ldots, K\right\}
$$

such that the set

$$
\left\{\varphi_{k}, k=1, \ldots, K\right\} \cup\left\{\psi_{j}, j=1, \ldots, J\right\}
$$

is a complete set of quasimodes for $P$.

Remark 7. Note that this lemma applies to sets of quasimodes that do not have positive density.

Proof. Let $u_{j}$ be eigenfunctions of $P$ corresponding to $E_{j}$. Select an energy $E$. Then, by the proof of Lemma 1, there are gaps at distance $c_{1}(h)$ of size $h^{d+1}$ in the spectrum of $P$ near $E$. Let

$$
\Lambda \stackrel{\text { def }}{=}\left\{u_{k}:\left|E_{k}-E\right| \leq c_{1}(h)\right\}
$$

and

$$
\Lambda^{\prime} \stackrel{\text { def }}{=}\left\{\psi_{j}:\left|E_{j}-E\right|=o\left(h^{d+1}\right)\right\} .
$$

Then, for $\psi_{j} \in \Lambda^{\prime}$, we have that

$$
\psi_{j}=\sum_{u_{k} \in \Lambda} c_{j k} u_{k}
$$

Now, define

$$
N \stackrel{\text { def }}{=}|\Lambda| \quad \text { and } \quad M \stackrel{\text { def }}{=}\left|\Lambda^{\prime}\right|
$$

letting

$$
b_{j}=\left(c_{j 1}, c_{j 2}, \ldots, c_{j N}\right), \quad j=1, \ldots, M .
$$

Let $\left\{e_{1}, \ldots, e_{m}\right\}$ be an orthonormal basis for span $\left(\left\{b_{j}: j=1, \ldots, M\right\}\right)$. Apply the Gram-Schmidt process to obtain an orthonormal basis $\left\{e_{1}, \ldots, e_{M}, v_{M+1}, \ldots, v_{N}\right\}$ of $\mathbb{R}^{N}$ where

$$
v_{k}=\left(v_{k 1}, \ldots, v_{k N}\right) .
$$


Then, letting

$$
\varphi_{k}=\sum_{j} v_{k j} u_{j}, \quad M+1 \leq k \leq N,
$$

$\left\{\psi_{1}, \ldots, \psi_{M}, \varphi_{M+1}, \ldots, \varphi_{N}\right\}$ is an almost orthonormal basis for span $\Lambda$. Repeating this process for each cluster, we obtain a complete set of quasimodes.

We also need the following restatement of results in [4], Section 4.3, that is found in [6], Lemma A1.

Lemma 5. Fix $T>0$. Assume that $A \in \Psi^{\mathrm{comp}}\left(M^{o}\right)$ is supported away from the boundary of $M$ and $\mathrm{WF}_{h}(A) \subset p^{-1}([a, b]) \backslash \mathcal{B}_{T}$. Then, for each $\chi \in C_{c}^{\infty}\left(M^{o}\right)$ and for each $t \in[-T, T]$, the operator $\chi e^{-i t P / h} A$ is a Fourier integral operator supported away from $\partial M$ and associated to the restriction of $\varphi_{t}$ to a neighborhood of $\mathrm{WF}_{h}(A) \cap \varphi_{t}^{-1}(\operatorname{supp} \chi)$, plus an $O_{L^{2} \rightarrow L^{2}}\left(h^{\infty}\right)$ remainder. The following version of Egorov's theorem holds:

$$
\chi e^{i t P / h} A e^{-i t P / h}=A_{t, \chi}+O\left(h^{\infty}\right)_{L^{2}(M) \rightarrow L^{2}(M)},
$$

where $A_{T, \chi} \in \Psi^{\mathrm{comp}}\left(M^{o}\right)$ is supported away from $\partial M$ and $\sigma_{h}\left(A_{T, \chi}\right)=\chi\left(a \circ \varphi_{t}\right)$.

Now, we prove Theorem 1, following [6], Appendix A.

Proof of Theorem 1. Let $\left(\psi_{j}, E_{j}\right)$ be a positive density set of quasimodes. By Lemma 4 , and the fact that our original quasimodes have positive density, we may assume without loss that $\left(\psi_{j}, E_{j}\right)$ is a complete set of quasimodes (see Definition 2).

We first show that

$$
\limsup _{h \rightarrow 0} h^{d} \sum_{E_{j} \in[a, b]}\left|\left\langle B \psi_{j}, \psi_{j}\right\rangle-f_{p^{-1}\left(E_{j}\right)} \sigma_{h}(B) d \mu_{E_{j}}\right|=0 .
$$

Then, by a standard diagonal argument that can be found, for example, in [23], Theorem 15.5, we extract the set $\Lambda(h)$.

Take $a^{\prime}, b^{\prime}$ such that $a^{\prime}<a<b<b^{\prime}$ and the assumptions (1.2) and (1.1) hold for $E \in\left[a^{\prime}, b^{\prime}\right]$. (If they do not hold when $E \notin[a, b]$, then we need to take $a^{\prime}, b^{\prime}$ getting close to $a$ and $b$. e.g. Let $a^{\prime}=a-1 / T$ and $b^{\prime}=b+1 / T$. Then estimate the extra contributions by the Weyl law.) Now, fix a $T>0$ and choose $\chi_{T} \in C_{c}^{\infty}\left(M^{o}\right)$ with $0 \leq \chi_{T} \leq 1$ and

$$
\int_{T^{*} M \cap p^{-1}\left(\left[a^{\prime}-1, b^{\prime}-1\right]\right)}\left(1-\chi_{T}\right)^{2} d \mu_{\sigma} \leq T^{-1} .
$$

Let $\psi \in C_{c}^{\infty}\left(a^{\prime}-1, b^{\prime}+1\right)$ have

$$
\psi(E) \int_{p^{-1}(E)} \chi_{T} d \mu_{E}=\int_{p^{-1}(E)} \sigma_{h}(B) d \mu_{E}, \quad E \in\left[a^{\prime}, b^{\prime}\right] .
$$


Then, by Lemma 3, it is enough to show that the conclusion holds for

$$
B-\psi(P(h)) \chi_{T},
$$

whose symbol integrates to 0 on $p^{-1}(E)$. Therefore, we assume, without loss, that

$$
\int_{p^{-1}(E)} \sigma_{h}(B) d \mu_{E}=0, \quad E \in\left[a^{\prime}, b^{\prime}\right] .
$$

By elliptic estimates, we may assume that $\mathrm{WF}_{h}(B) \subset p^{-1}\left(\left(a^{\prime}, b^{\prime}\right)\right)$. More specifically, $B \in \Psi^{\text {comp }}$. Thus, we can write $B=B_{T}^{\prime}+B_{T}^{\prime \prime}$, where $\mathrm{WF}_{h}\left(B_{T}^{\prime}\right) \cap \mathcal{B}_{T}=\varnothing$ and $\left\|\sigma_{h}\left(B_{T}^{\prime \prime}\right)\right\|_{L^{2}\left(p^{-1}\left[a^{\prime}, b^{\prime}\right]\right)} \leq T^{-1}$.

Then, by Hölder's inequality, Lemma 2 and [6], Lemma 2.2, we have that

$$
\begin{aligned}
h^{d} \sum_{E_{j} \in[a, b]}\left|\left\langle B_{T}^{\prime \prime} \psi_{j}, \psi_{j}\right\rangle\right| & \leq C\left(h^{d} \sum_{E_{j} \in[a, b]}\left\|B_{T}^{\prime \prime} \psi_{j}\right\|^{2}\right)^{1 / 2} \\
& =C\left(h^{d} \sum_{E_{j} \in[a, b]}\left\|B_{T}^{\prime \prime} u_{j}\right\|^{2}\right)^{1 / 2}+o(1) \\
& \leq C\left\|\sigma_{h}\left(B_{T}^{\prime \prime}\right)\right\|_{L^{2}\left(p^{-1}\left[a^{\prime}, b^{\prime}\right]\right)}+o(1) .
\end{aligned}
$$

Hence, the contribution of $B_{T}^{\prime \prime}$ goes to 0 in the $\operatorname{limit}_{T \rightarrow \infty} \lim _{T \rightarrow p_{h \rightarrow 0}}$ and we may replace $B$ by $B_{T}^{\prime}$.

Now, by Duhamel's formula and the unitarity of $e^{i t P / h}$,

$$
e^{i t P / h} \psi_{j}=e^{i t E_{j} / h} \psi_{j}+\frac{i}{h} \int_{0}^{t} e^{i(t-s) P / h} o\left(h^{2 d+1}\right)=e^{i t E_{j} / h} \psi_{j}+o_{T}\left(h^{2 d}\right) .
$$

So, defining

$$
\langle A\rangle_{T} \stackrel{\text { def }}{=} \frac{1}{T} \int_{0}^{T} e^{i t P / h} A e^{-i t P / h} d t,
$$

and using Lemma 2 and Hölder's inequality, we have

$$
\begin{aligned}
h^{d} \sum_{E_{j} \in[a, b]}\left|\left\langle B_{T}^{\prime} \psi_{j}, \psi_{j}\right\rangle\right| & =h^{d} \sum_{E_{j} \in[a, b]}\left|\left\langle B_{T}^{\prime} e^{-i t E_{j} / h} \psi_{j}, e^{-i t E_{j} / h} \psi_{j}\right\rangle\right| \\
& =h^{d} \sum_{E_{j} \in[a, b]}\left|\left\langle\left\langle B_{T}^{\prime}\right\rangle_{T} \psi_{j}, \psi_{j}\right\rangle\right|+o_{T}\left(h^{2 d}\right) \\
& \leq\left(h^{d} \sum_{E_{j} \in[a, b]}\left(\left\|\left\langle B_{T}^{\prime}\right\rangle_{T} \psi_{j}\right\|+o_{T}\left(h^{2 d}\right)\right)^{2}\right)^{1 / 2} \\
& =\left(h^{d} \sum_{E_{j} \in[a, b]}\left\|\left\langle B_{T}^{\prime}\right\rangle_{T} u_{j}\right\|^{2}\right)^{1 / 2}+o_{T}(1) .
\end{aligned}
$$


From this point forward, the proof is identical to that in [6], Theorem A.2. By Lemma 5, $\left\langle B_{T}^{\prime}\right\rangle_{T} \chi_{T}$ is, up to an $O\left(h^{\infty}\right)_{L^{2} \rightarrow L^{2}}$ remainder, a pseudodifferential operator in $\Psi^{\text {comp }}$ compactly supported inside $M^{o}$ and with principal symbol

$$
\sigma_{h}\left(\left\langle B_{T}^{\prime}\right\rangle_{T} \chi_{T}\right)=\frac{\chi_{T}}{T} \int_{0}^{T} \sigma_{h}\left(B_{T}^{\prime}\right) \circ \varphi_{t} d t .
$$

Now, all that remains to show is

$$
\lim _{T \rightarrow \infty} \limsup _{h \rightarrow 0} h^{d} \sum_{E_{j} \in[a, b]}\left\|\left\langle B_{T}^{\prime}\right\rangle_{T} u_{j}\right\|^{2}=0 .
$$

Since, by Lemma 3 ,

$$
\limsup _{h \rightarrow 0}(2 \pi h)^{d} \sum_{E_{j} \in[a, b]}\left\|\left(1-\chi_{T}\right) u_{j}\right\|_{L^{2}}^{2} \leq T^{-1},
$$

we can replace $\left\langle B_{T}^{\prime}\right\rangle_{T}$ by $\left\langle B_{T}^{\prime}\right\rangle_{T}$. Thus, by [6], Lemma 2.2, it remains to show that

$$
\lim _{T \rightarrow \infty}\left\|\sigma_{h}\left(\left\langle B_{T}^{\prime}\right\rangle_{T} \chi_{T}\right)\right\|_{L^{2}\left(p^{-1}\left(\left[a^{\prime}, b^{\prime}\right]\right)\right)} \leq \lim _{T \rightarrow \infty}\left\|\left\langle\sigma_{h}\left(B_{T}^{\prime}\right)\right\rangle_{T}\right\|_{L^{2}\left(p^{-1}\left(\left[a^{\prime}, b^{\prime}\right]\right)\right)}=0 .
$$

To do this, write

$$
\left\|\left\langle\sigma_{h}\left(B_{T}^{\prime}\right)\right\rangle_{T}\right\|_{L^{2}\left(p^{-1}(E)\right)} \leq\left\|\left\langle\sigma_{h}(B)\right\rangle_{T}\right\|_{L^{2}\left(p^{-1}(E)\right)}+\left\|\left\langle\sigma_{h}\left(B_{T}^{\prime \prime}\right)\right\rangle_{T}\right\|_{L^{2}\left(p^{-1}(E)\right)} .
$$

The first term on the right goes to 0 when $T \rightarrow \infty$ by the von Neumann ergodic theorem and the second term is bounded by $\left\|\sigma_{h}\left(B_{T}^{\prime \prime}\right)\right\|_{L^{2}\left(p^{-1}(E)\right)}$ and hence also goes to 0 .

Remark 8. Notice that (2.3) is the only step in which the ergodicity of the flow is used. This will be important when we adapt the result to ergodic invariant subsets of phase space.

\section{Quantum ergodicity for subsets}

Now, we prove Theorem 2 using the fact that only step (2.3) uses the ergodicity of the flow. For completeness, we include the following elementary lemma.

Lemma 6. Let $U$ satisfy (1.4), let $\mu_{2}$ be a finite measure on $p^{-1}(E)$ and suppose that for all $a \in C^{\infty}\left(p^{-1}(E)\right)$ compactly supported away from $\partial M$ with

$$
\int_{U \cap p^{-1}(E)} a d \mu_{E}=0,
$$


we have

$$
\int_{U \cap p^{-1}(E)} a d \mu_{2}=0
$$

Then,

$$
\left.\left.\mu_{2}\right|_{U \cap p^{-1}(E)} \equiv c \mu_{E}\right|_{U \cap p^{-1}(E)}
$$

for some $c \geq 0$.

Proof. Let

$$
V=U \cap p^{-1}(E)
$$

and

$$
\mu_{1}=\left(\mu_{E}(V)\right)^{-1} \mu_{E} ;
$$

then, $\mu_{1}(V)=1$. Define $\chi \in C^{\infty}\left(p^{-1}(E)\right)$ compactly supported away from $\partial M$ with

$$
0 \leq \chi \leq 2, \quad \int_{V} \chi d \mu_{1}=1, \quad \int_{V} \chi d \mu_{2}=c_{2}>0 .
$$

(To see that such functions exist simply take non-negative approximations to $1_{V}$ with support inside $M^{o}$.)

Now, let $a \in C^{\infty}\left(p^{-1}(E)\right)$ compactly supported away from $\partial M$ with

$$
\int_{V} a d \mu_{1}=\bar{a}
$$

Then,

$$
\int_{V} a-\bar{a} \chi d \mu_{1}=0
$$

and hence

$$
\int_{V} a-\bar{a} \chi d \mu_{2}=0
$$

Therefore, for $a \in C^{\infty}\left(p^{-1}(E)\right)$ compactly supported away from $\partial M$,

$$
\int_{V} a d \mu_{2}=c_{2} \int_{V} a d \mu_{1}
$$

But, $\left.\mu_{1}\right|_{V}$ and $\left.\mu_{2}\right|_{V}$ are positive distributions of order 0 since $V$ satisfies (1.4). Hence, since

$$
\int_{V} a d \mu_{2}=\int_{V} a d\left(c_{2} \mu_{1}\right)=0
$$

for all $a \in C^{\infty}\left(p^{-1}(E)\right)$ compactly supported away from $\partial M$, and $M$ can be exhausted by compact subsets, $\left.\left.\mu_{2}\right|_{V} \equiv c_{2} \mu_{1}\right|_{V}$. 
Proof of Theorem 2. Lemma 4 shows that without loss of generality we may work with $\psi_{j}$ forming a complete set of quasimodes. Fix $A \in \Psi_{h}^{0}(M)$ with symbol $a=\sigma_{h}(A)$ compactly supported away from $\partial M$ satisfying

$$
\int_{U \cap p^{-1}(E)} a d \mu_{E}=0, \quad E \in[a, b] .
$$

Fix $\varepsilon>0$. Then, let $U_{\varepsilon}$ be open with

$$
\bar{U} \subset U_{\varepsilon}
$$

and

$$
\mu_{E}\left(\left(U_{\varepsilon} \backslash U\right) \cap p^{-1}(E)\right)<\varepsilon, \quad E \in[a, b] .
$$

(Note that we use (1.4) here.) Let $\chi_{\varepsilon} \in C_{c}^{\infty}\left(U_{\varepsilon}\right)$ compactly supported away from $\partial M$ with $\left.\chi_{\varepsilon}\right|_{\bar{U}} \equiv 1,0 \leq \chi_{\varepsilon} \leq 1$. Then, let $a_{\varepsilon}=\chi_{\varepsilon} a$. Based on the arguments used to prove Theorems 1, we have that

$$
\limsup _{h \rightarrow 0} h^{d} \sum_{E_{j} \in[a, b]}\left|\left\langle A_{\varepsilon} \psi_{j}, \psi_{j}\right\rangle\right| \leq C\left\|\left\langle a_{\varepsilon}\right\rangle_{T}\right\|_{L^{2}\left(p^{-1}[a, b]\right)},
$$

where $A_{\varepsilon}=a_{\varepsilon}(x, h D)$,

$$
\left\langle a_{\varepsilon}\right\rangle_{T} \stackrel{\text { def }}{=} \frac{1}{T} \int_{0}^{T} a_{\varepsilon} \circ \varphi_{t}(x, \xi) d t .
$$

We have that $\varphi_{t}$ is ergodic on $U$ and $U$ is invariant under $\varphi_{t}$. Hence, by the von Neumann ergodic theorem

$$
\left\langle a_{\varepsilon} 1_{U}\right\rangle_{T} \rightarrow f_{U} a_{\varepsilon} d \mu_{E}=0 \quad \text { in } L^{2}\left(p^{-1}(E)\right) .
$$

Again, by the ergodic theorem, $\left\langle a_{\varepsilon} 1_{U^{c}}\right\rangle_{T} \rightarrow P a_{\varepsilon}$ in $L^{2}\left(p^{-1}(E)\right)$ with $\left\|P a_{\varepsilon}\right\|_{L^{2}} \leq$ $C\|a\|_{L^{\infty} \varepsilon}$. Hence,

$$
\lim _{T \rightarrow \infty}\left\|\left\langle a_{\varepsilon}\right\rangle_{T}\right\|_{L^{2}\left(p^{-1}[a, b]\right)} \leq C\|a\|_{L^{\infty} \varepsilon} .
$$

We now show that there exists a full density subset

$$
\Lambda(h) \subset\left\{\left(\psi_{j}, E_{j}\right) \mid a \leq E_{j} \leq b\right\}
$$

such that for all $\psi_{j} \in \Lambda(h)$ and all $A \in \Psi_{h}^{0}$ with symbol $a=\sigma_{h}(A)$ satisfying (3.1)

$$
\lim _{\varepsilon \rightarrow 0} \lim _{h \rightarrow 0}\left\langle A_{\varepsilon} \psi_{j}, \psi_{j}\right\rangle=0 .
$$

We first do this for one such $a \in C^{\infty}\left(T^{*} M\right)$. Fix $\varepsilon>0$ and let

$$
\Gamma(h, \varepsilon)=\left\{a \leq E_{j} \leq b:\left|\left\langle A_{\varepsilon} \psi_{j}, \psi_{j}\right\rangle\right| \geq\left(C\|a\|_{L^{\infty \varepsilon}}\right)^{\frac{1}{2}}\right\} .
$$


Then, by the Chebyshev inequality, (3.2), and (3.3),

$$
h^{d}|\Gamma(h, \varepsilon)| \leq\left(C \varepsilon\|a\|_{L^{\infty}}\right)^{\frac{1}{2}}
$$

and for $E_{j} \notin \Gamma(h)$

$$
\left|\left\langle A_{\varepsilon} \psi_{j}, \psi_{j}\right\rangle\right| \leq\left(C \varepsilon\|a\|_{L^{\infty}}\right)^{\frac{1}{2}}
$$

But, by the Weyl law,

$$
\begin{aligned}
|\Gamma(h, \varepsilon)| /\left|\left\{a \leq E_{j} \leq b\right\}\right| & =h^{d}|\Gamma(h, \varepsilon)| /\left(\operatorname{Vol}\left(p^{-1}[a, b]\right)+o(1)\right) \\
& \leq C\left(\|a\|_{L} \infty \varepsilon\right)^{1 / 2}+o(1) .
\end{aligned}
$$

Now, let $\varepsilon_{m} \rightarrow 0$ and define

$$
\Gamma(h)=\bigcap_{m} \Gamma\left(h, \varepsilon_{m}\right) .
$$

Then, $\Gamma(h)$ has 0 density as $h \rightarrow 0$. Hence,

$$
\Lambda(h)=\left\{a \leq E_{j} \leq b\right\} \backslash \Gamma(h)
$$

has full density as $h \rightarrow 0$ and has the desired property for the operator $a(x, h D)$.

To complete the construction of $\Lambda(h)$, first observe that it is enough to consider $A \in$ $\Psi_{h}^{-\infty}$ since $\psi_{j}$ are microlocalized in $p^{-1}[a, b]$. Then, take a countable dense set, $\left\{A_{k}\right\}$ of $\Psi_{h}^{-\infty} \cap\left\{A \in \Psi_{h}^{0}:\left\|\sigma_{h}(A)\right\|_{L^{\infty}} \leq 1, \sigma_{h}(A)\right.$ satisfies (3.1) $\}$ and apply a variant of the standard diagonal argument (contained for example, in [23], Theorem 15.5).

Now, suppose that $\left\{\psi_{j}\right\} \subset \Lambda(h)$ is a further subset with defect measure $\mu$. Then, for $A \in \Psi_{h}^{0}$ with symbol satisfying (3.1), we have that

$$
\lim _{h \rightarrow 0}\left\langle A_{\varepsilon} \psi_{j}, \psi_{j}\right\rangle=\int \sigma_{h}(A)_{\varepsilon} d \mu=o(1) .
$$

Hence, by the dominated convergence theorem,

$$
\int_{U} \sigma_{h}(A) d \mu=0
$$

Since $\left\{\psi_{j}\right\}$ is a subset of a positive density set of quasimodes on $[a, b]$, there exists $E \in[a, b]$ such that $\operatorname{supp} \mu \subset p^{-1}(E)$. Thus, we may apply Lemma 6 to obtain the result. 


\section{From semiclassical to standard quantum ergodicity}

For completeness and to present the proof in the quasimode case, we will now pass from Theorem 1 with $P=-h^{2} \Delta$ to Theorem 3 .

Proof of Theorem 3. Let $h^{2} \lambda_{j}^{2}=E_{j}$ and $\lambda=h^{-1}, \chi \in C^{\infty} \chi(\xi) \equiv 0$ for $|\xi| \leq 1$ and $\chi \equiv 1$ for $|\xi| \geq 2$, and $\chi_{\varepsilon}=\chi(\xi / \varepsilon)$.

Let $\hat{A}$ be a homogeneous pseudodifferential operator of order 0 on $M$ with symbol compactly supported away from $\partial M$. Define

$$
a_{0} \stackrel{\text { def }}{=} \sigma(\widehat{A}) .
$$

Then, $a_{0}$ is homogeneous degree 0 on $T^{*} M \backslash\{0\}$. Hence

$$
a_{0}(x, D) \chi(D)=a_{0}(x, h D) \chi(h D / h) .
$$

Now, define $A_{\varepsilon} \in \Psi^{0}\left(M^{o}\right)$ by

$$
A_{\varepsilon} \stackrel{\text { def }}{=} a(x, h D) \chi_{\varepsilon}(h D) .
$$

Theorem 1 gives, for $0<a<b$,

$$
h^{d} \sum_{h \lambda_{j} \in[a, b]}\left|\left\langle A_{\varepsilon} \psi_{j}, \psi_{j}\right\rangle-f_{p^{-1}\left(E_{j}\right)} \sigma_{h}\left(A_{\varepsilon}\right) d \mu_{E_{j}}\right| \longrightarrow 0, \quad h=\lambda^{-1} \rightarrow 0 .
$$

Since $\sigma_{h}\left(A_{\varepsilon}\right)=a_{0}(x, \xi) \chi_{\varepsilon}(\xi)$ and $a_{0}$ is homogeneous degree 0 , we have

$$
f_{p^{-1}\left(E_{j}\right)} \sigma_{h}\left(A_{\varepsilon}\right) d \mu_{E_{j}}=f_{S^{*} M} \sigma(\widehat{A}) d \mu_{L}+O(\varepsilon) .
$$

Hence, we need to show,

$$
\lim _{\varepsilon \rightarrow 0} \limsup _{h \rightarrow 0} h^{d} \sum_{h \lambda_{j} \in[a, b]}\left\langle\left(\hat{A}-A_{\varepsilon}\right) \psi_{j}, \psi_{j}\right\rangle=0 .
$$

By Hölder's inequality, Lemma 2, and [6], Lemma 2.2,

$$
\begin{aligned}
& h^{d} \sum_{h \lambda_{j} \in[a, b]}\left|\left\langle\left(\hat{A}-A_{\varepsilon}\right) \psi_{j}, \psi_{j}\right\rangle\right| \\
& \quad \leq C\left(h^{d} \sum_{h \lambda_{j} \in[a, b]}\left\|\left(\hat{A}-A_{\varepsilon}\right) \psi_{j}\right\|^{2}\right)^{1 / 2} \\
& \quad=C\left(h^{d} \sum_{h \lambda_{j} \in[a, b]}\left\|\left(\hat{A}-A_{\varepsilon}\right) u_{j}\right\|^{2}\right)^{1 / 2}+o(1) \\
& \quad \leq C\left\|\sigma_{h}\left(\hat{A}-A_{\varepsilon}\right)\right\|_{L^{2}\left(p^{-1}([a, b])\right)}+o(1) .
\end{aligned}
$$


We observe

$$
\lim _{\varepsilon \rightarrow 0}\left\|\sigma_{h}\left(\widehat{A}-A_{\varepsilon}\right)\right\|_{L^{2}\left(p^{-1}([a, b])\right)}=0 .
$$

Thus, for any $\delta>0$, we have

$$
\limsup _{h \rightarrow 0} h^{d} \sum_{h \lambda_{j} \in[\delta, 1]}\left|\left\langle\widehat{A} \psi_{j}, \psi_{j}\right\rangle-f_{S^{*} M} \sigma(\widehat{A}) d \mu_{L}\right|=0 .
$$

All that remains is to show that

$$
\lim _{\delta \rightarrow 0} \limsup _{h \rightarrow 0} h^{d} \sum_{h \lambda_{j} \in[0, \delta)}\left|\left\langle\widehat{A} \psi_{j}, \psi_{j}\right\rangle-f_{S^{*} M} \sigma(\widehat{A}) d \mu_{L}\right|=0 .
$$

Letting

$$
\bar{a}_{0}=\int_{S^{*} M} \sigma(\widehat{A}) d \mu_{L},
$$

and applying the Weyl law, and the Hölder's inequality we get

$$
\begin{aligned}
h^{d} \sum_{h \lambda_{j} \in[0, \delta)}\left|\left\langle\widehat{A} \psi_{j}, \psi_{j}\right\rangle-\bar{a}_{0}\right| & \leq C\left(h^{d} \sum_{h \lambda_{j} \in[0, \delta)}\left\|\left(\widehat{A}-\bar{a}_{0}\right) \psi_{j}\right\|^{2}\right)^{1 / 2} \\
& =O\left(\delta^{d}\right)\left\|\left(\hat{A}-\bar{a}_{0}\right)\right\|_{L^{2} \rightarrow L^{2}}+O_{\delta}(h) .
\end{aligned}
$$

Once again, to obtain the full density subsequence, we employ a standard diagonal argument.

We may also make similar arguments to those above to pass to the homogeneous setting for Theorem 2. In this case, we obtain Theorem 4.

Proof of Theorem 4. Let

$$
\widehat{U} \stackrel{\text { def }}{=}\left\{(x, \xi) \in T^{*} M:(x, \xi /|\xi|) \in U\right\} .
$$

Then, $\widehat{U}$ satisfies (1.4) and (1.5) for any $0<a<b$. Hence, by Theorem 2 there exists

$$
\Lambda_{\delta}(h) \subset\left\{h \lambda_{j} \in[\delta, 1]\right\}
$$

of full density such that for all $\left\{\psi_{j}\right\} \subset \Lambda_{\delta}(h)$ with defect measure $\mu$, there is an $E \in[\delta, 1]$ such that

$$
\operatorname{supp} \mu \subset S_{E}^{*} M \quad \text { and }\left.\left.\quad \mu\right|_{\left.\hat{U} \cap S_{E}^{*} M\right)} \equiv c \mu_{E}\right|_{\hat{U} \cap S_{E}^{*} M},
$$

where

$$
S_{E}^{*} M=\left\{(x, \xi) \in T^{*} M:|\xi|=E\right\}
$$


Now, for $a \in C^{\infty}\left(T^{*} M \backslash\{0\}\right)$, homogeneous of degree $0, \varepsilon>0$ small enough and $\chi_{\varepsilon}$ as in the proof of Theorem 3

$$
\int_{\widehat{U} \cap S_{E}^{*} M} a \chi_{\varepsilon} d \mu_{E}=\int_{\widehat{U} \cap S_{E}^{*} M} a d \mu_{E}=\int_{U} a d \mu_{L}
$$

and hence the homogeneous defect measure, $\mu$ of has $\left.\left.\mu\right|_{U} \equiv c \mu_{L}\right|_{U}$.

But, by the Weyl Law, $h^{d}\left|\left\{h \lambda_{j} \in[0, \delta)\right\}\right|=O\left(\delta^{d}\right)$. Thus, letting

$$
\Lambda(h)=\bigcup_{\delta>0} \Lambda_{\delta}(h),
$$

we have that $\Lambda(h) \subset\left\{h \lambda_{j} \in[0,1]\right\}$ has full density and for all $\left\{\psi_{j}\right\}$ subsequences of $\Lambda(h)$ with homogeneous defect measure $\mu$, there exists $c \geq 0$ with $\left.\mu\right|_{U}=\left.c \mu_{L}\right|_{U}$.

\section{An example}

We now present an example to which Theorem 4 applies. Let $\Omega$ be a symmetric mushroom billiard, as in [3], composed of a hat that is a semicircle of radius 1 and a base that has width $w$ and height $h$ (Figure 1 shows such a mushroom with two billiard flows). Then $S^{*} \Omega$ has a subset $U$ satisfying (1.4) and (1.5); see [3]. Let

$$
u_{n k}=\sin (n \theta) J_{n}\left(\alpha_{n, k} r\right)
$$

be the eigenfunctions of the Dirichlet Laplacian on the semidisk. Here $J_{n}$ denotes the Bessel function of the first kind of order $n$ and $\alpha_{n, k}$ the $k^{\text {th }}$ positive zero of $J_{n}$.

From [15], Appendix A, we have that for $0<\gamma<\frac{2}{3}, z \in\left(0,1-n^{\gamma-\frac{2}{3}}\right)$

$$
0<J_{n}(n z)<2^{-n^{\gamma / 3}} .
$$

Also, from [16], we have for $0 \leq k<c_{2} n$, that $n<\alpha_{n, k}<C n$ for some $C>0$. Thus, $J_{n}\left(\alpha_{n, k} r\right)=O\left(2^{-n^{\gamma / 3}}\right)$ for $r<\frac{1}{2 C}$.

Now, suppose $0<w<\frac{1}{4 C}$. Then, let $\chi \in C^{\infty}(\Omega)$ with $\chi \equiv 0$ in $|r|<\frac{1}{4 C}$ and $\chi \equiv 1$ in $|r| \geq \frac{1}{2 C}$. If we let

$$
v_{n, k} \stackrel{\text { def }}{=} \chi u_{n, k},
$$

for $0 \leq k<c_{2} n$, and extend by 0 outside of the hat of $\Omega$, then $v_{n, k}$ are quasimodes for the Dirichlet Laplacian on $\Omega$ with

$$
\left(-\Delta-\alpha_{n, k}^{2}\right) v_{n, k}=O\left(2^{-n^{\gamma / 3}}\right)
$$


Now, by the orthogonality of $u_{n, k}, v_{n, k}$ are orthogonal up to $O\left(e^{-C n^{\gamma / 3}}\right)$. Hence, $\left\{v_{n, k}\right\}$ are a family of $\frac{c_{2} n\left(c_{2} n+1\right)}{2}$ almost orthogonal quasimodes with $O\left(e^{-C n^{\gamma / 3}}\right)$ error. Thus,

$$
\left\{\left(v_{n, k}, \alpha_{n, k}^{2}\right), 0 \leq k<c_{2} n, n=1, \ldots\right\}
$$

form a positive density set of quasimodes for $-\Delta$ on $[0,1]$.

Since the $v_{n, k}$ are $O\left(n^{-\infty}\right)$ quasimodes, $\mathrm{WF}_{h}\left(v_{n, k}\right)$ is invariant under the Hamiltonian flow (see, for example, [23], Section 12.3). Therefore, since $\mathrm{WF}_{h}\left(v_{n, k}\right)$ does not intersect the foot of $\Omega$, the quasimodes must concentrate away from the ergodic set, $U$. Thus, for any subsequence of $\left\{v_{n, k}\right\}$ with a defect measure $\mu,\left.\mu\right|_{U} \equiv 0$. Hence, Theorem 2 applies and the constant we obtain is 0 .

\section{References}

[1] N. Anantharaman and G. Riviére. Dispersion and controllability for the Schrödinger equation on negatively curved manifolds Anal. PDE 5 (2012), 313-338. MR 2970709 Zbl 1267.35176

[2] A. H. Barnett and T. Betcke, Quantum mushroom billiards. Chaos 17 (2007), Article Id. 043125. MR 2380041 Zbl 1163.37310

[3] L. A. Bunimovich, Mushrooms and other billiards with divided phase space. Chaos 11 (2001), 802-808. MR 1875161 Zbl 1080.37582

[4] H. Christianson, Quantum monodromy and nonconcentration near a closed semihyperbolic orbit. Trans. Amer. Math. Soc. 363 (2011), 3373-3438. MR 2775812 Zbl 1230.58020

[5] Y. Colin de Verdiére, Ergodicité et fonctions propres du laplacien. Comm. Math. Phys. 102 (1985), 497-502. MR 0818831 Zbl 0592.58050

[6] S. Dyatlov and M. Zworski, Quantum ergodicity for restrictions to hypersurfaces. Nonlinearity 26 (2013), 35-52. MR $3001760 \mathrm{Zbl} 06163460$

[7] P. Gérard and E. Leichtnam, Ergodic properties of eigenfunctions for the Dirichlet problem. Duke Math. J. 71 (1993), 559-607. MR 1233448 Zbl 0788.35103

[8] A. Hassell, Ergodic billiards that are not quantum unique ergodic. With an appendix by the author and L. Hillairet. Ann. of Math. (2) 171 (2010), 605-619. MR 2630052 Zbl 1196.58014

[9] B.Hellfer, A. Martinez, and D. Robert, Ergodicité et limite semi-classique. Comm. Math. Phys. 109 (1987), 313-326. MR 0880418 Zbl 0624.58039

[10] L. Hörmander, The analysis of linear partial differential operators. III. Pseudodifferential operators. Grundlehren der Mathematischen Wissenschaften 274. Springer Verlag, Berlin, 1985. Reprint, Classics in Mathematics. Springer Verlag, Berlin, 2007. MR 0781536 MR 2304165 (reprint) Zbl 0601.35001 Zbl 1115.35005 (reprint)

[11] V. F. Lazutkin, KAM theory and semiclassical approximations to eigenfunctions. With an addendum by A. I. Shnirelman. Ergebnisse der Mathematik und ihrer Grenzgebiete (3) 24. Springer Verlag, Berlin etc., 1993. MR 1239173 Zbl 0814.58001 
[12] J. Marklof. Quantum Leaks. Comm. Math. Phys. 264 (2006), 303-316. MR 2215606 Zbl 1233.58004

[13] J. Marklof and S. O'Keefe. Weyl's law and quantum ergodicity for maps with divided phase space. With an appendix "Converse quantum ergodicity" by Steve Zelditch. Nonlinearity 18 (2005), 277-304. MR 2109478 Zbl 1109.81037

[14] J. Marklof and Z. Rudnick, Almost all eigenfunctions of a rational polygon are uniformly distributed. J. Spectr. Theory 2 (2012), 107-113. MR 2879311 Zbl 1242.37027

[15] B. T. Nguyen and D. S. Grebenkov. Localization of Laplacian eigenfunctions in circular, spherical and elliptical domains. SIAM J. Appl. Math. 73 (2013), 780-803. MR 3038118 Zbl 06190521

[16] F. W. J. Olver, The asymptotic expansion of Bessel functions of large order. Philos. Trans. Roy. Soc. London. Ser. A. 247 (1954), 328-368. MR 0067250 Zbl 0070.30801

[17] G. Riviére, Remarks on quantum ergodicity. J. Mod. Dyn. 7 (2013), 119-133. MR 3071468 Zbl 06174323

[18] I. C. Percival, Regular and irregular spectra. J. Phys. B: At. Mol. Phys. 6(1973), L229-232.

[19] A. Shnirelmann, Ergodic properties of eigenfuncions. Usp. Math. Nauk. 29 (1974), 181-182.

[20] S. Zelditch, Note on quantum unique ergodicity. Proc. Amer. Math. Soc. 132 (2004), 1869-1872. MR 2051153 Zbl 1055.58016

[21] S. Zelditch, Uniform distribution of eigenfunctions on compact hyperbolic surfaces. Duke Math. J. 55 (1987) 919-941. MR 0916129 Zbl 0643.58029

[22] S. Zelditch and M. Zworski, Ergodicity of eigenfunctions for ergodic billiards. Comm. Math. Phys. 175 (1996), 673-682. MR 1372814 Zbl 0840.58048

[23] M. Zworski, Semiclassical analysis. Graduate Studies in Mathematics 138. American Mathematical Society, Providence, RI, 2012. MR 2952218 Zbl 1252.58001

Received September 24, 2012; revised January 16, 2013

Jeffrey Galkowski, Mathematics Department, University of California, Berkeley, CA 94720, U.S.A.

E-mail: jeffrey.galkowski@math.berkeley.edu 